\title{
Transmissible Mitochondrial Hypovirulence in a Natural Population of Cryphonectria parasitica
}

\author{
Dipnath Baidyaroy, ${ }^{1}$ David H. Huber, ${ }^{1}$ Dennis W. Fulbright, ${ }^{1}$ and Helmut Bertrand ${ }^{2}$ \\ ${ }^{1}$ Department of Botany and Plant Pathology, Michigan State University, East Lansing 48824-1312, U.S.A.; \\ ${ }^{2}$ Department of Microbiology, Michigan State University, East Lansing 48824-1101, U.S.A. \\ Accepted 27 September 1999.
}

\begin{abstract}
A cytoplasmically transmissible hypovirulence syndrome has been identified in virus-free strains of the chestnut blight fungus Cryphonectria parasitica isolated from healing cankers on American chestnut trees in southwestern Michigan. The syndrome is associated with symptoms of fungal senescence, including a progressive decline in the growth potential and abundance of conidia, and elevated levels of respiration through the cyanide-insensitive alternative oxidase pathway. Conidia from senescing mycelia exhibited varying degrees of senescence ranging from normal growth to death soon after germination. Cytoplasmic transmission of hypovirulence between mycelia occurred by hyphal contact and coincided with the transfer of a specific restriction fragment length polymorphism from the mitochondrial DNA (mtDNA) of the donor strains into the mtDNA of virulent recipients. The transmission of the senescence phenotype was observed not only among vegetatively compatible strains but also among incompatible strains. Hypovirulence was present in isolates from the same location with different nuclear genotypes as identified by DNA fingerprinting. This study confirms that mitochondrial hypovirulence can occur spontaneously and spread within a natural population of a phytopathogenic fungus.
\end{abstract}

Additional keywords: cyanide-resistant respiration, KFC9, vegetative incompatibility, vic.

Several different infectious and debilitating, cytoplasmic factors can effectively reduce the aggressiveness of plantpathogenic, filamentous fungi (Buck 1986). In particular, infection by mycoviruses of Cryphonectria parasitica (Nuss 1992), Helminthosporium victoriae (Ghabrial 1988), and Rhizoctonia solani (Lakshman and Tavantzis 1994) have been shown to cause reduced virulence. In Ophiostoma ulmi, the cytoplasmic d-factor has been shown to be associated with reduced virulence (Brasier 1983; Rogers et al. 1986).

Corresponding author: Helmut Bertrand; Fax: 517-353-8957; E-mail: mhelmut@pilot.msu.edu

Present address of David H. Huber: Department of Biochemistry and Biophysics, University of Rochester School of Medicine and Dentistry, Rochester, NY 14642.

The first two authors have contributed equally to this work.
Cytoplasmically transmissible, reduced aggressiveness phenotypes in the chestnut blight fungus Cryphonectria parasitica have been termed "hypovirulence" and have resulted in the recovery of chestnut trees in nature. These hypovirulent phenotypes are primarily caused by infection of the fungus by double-stranded RNA (dsRNA) viruses (hypoviruses) (reviewed in Nuss 1992), which have specific effects on fungal pathogenicity by interfering with G-protein signaling pathways (Nuss 1996). However, some hypovirulent strains isolated from healing cankers do not contain any virus (Mahanti et al. 1993; Monteiro-Vitorello et al. 1995). Most of the virus-free hypovirulent strains of $C$. parasitica, when tested for respiration, show high levels of alternative oxidase activity (Mahanti et al. 1993; Monteiro-Vitorello et al. 1995), which normally is not exhibited by either wild-type strains or hypovirulent strains containing dsRNA viruses. This unique phenotype is singularly important in differentiating hypovirulent strains that lack viruses from those that contain viruses. The fact that the alternative oxidase-mediated pathway of respiration is induced in the dsRNA-free hypovirulent strains and that the attenuated state is transmitted cytoplasmically (Monteiro-Vitorello et al. 1995) suggests that this type of hypovirulence is associated with genetic alterations in the mitochondria that cause deficiencies in cytochrome-mediated respiration. The phenotypic characteristics of this dsRNA-free type of hypovirulence (Mahanti et al. 1993; MonteiroVitorello et al. 1995) suggest that it is comparable to debilitating phenotypes caused by mitochondrial DNA (mtDNA) mutations in Neurospora, Podospora, and Aspergillus spp. (reviewed in Griffiths 1992).

To test whether mtDNA mutations can indeed cause a cytoplasmically transmissible, hypovirulence phenotype in $C$. parasitica, Monteiro-Vitorello et al. (1995) artificially induced mutations in the mitochondrial chromosome of the virulent, wild-type strain Ep155. The mutant strains were found to have elevated levels of alternative oxidase activity, which is symptomatic of blockages in the cytochrome-mediated respiration pathway (Lambowitz and Slayman 1971; Lambowitz and Zannoni 1978; Vanlerberghe and McIntosh 1997) and were significantly reduced in virulence. That the alternative oxidase activity in these strains was induced because of a defect in the mitochondrial electron-transport system was confirmed by the finding that the mutants were deficient in cytochromes $a$ and $b$. The mutant (hypovirulence) phenotype was also cytoplasmically transmissible among vegetatively compatible strains of C. parasitica; hence, it appears to have infectious proper- 
ties similar to those associated with viral hypovirulence syndromes. Thus, it was established that mtDNA mutations could cause a debilitating disease similar to that encountered in virus-free, hypovirulent strains of Cryphonectria spp. isolated from healing cankers on trees. Hence the syndrome has been called "mitochondrial hypovirulence" to distinguish it from virus-mediated hypovirulence (Monteiro-Vitorello et al. 1995). However, the genetic basis of the naturally occurring, mitochondrial hypovirulence in virus-free $C$. parasitica strains remains to be elucidated.

Hypovirulent strains of Cryphonectria that do not contain detectable levels of dsRNA virus have been isolated from healing cankers of American chestnut trees in the Kellogg Forest, Augusta, Michigan (Mahanti et al. 1993). In this study, one isolate (KFC9) from that location has been characterized to determine the genetic basis of the hypovirulence trait that has arisen spontaneously in nature. The results indicate that this novel type of hypovirulence is associated not only with senescence and elevated levels of cyanide-resistant respiration, but also with a characteristic modification in the mitochondrial chromosome that is transmitted concordantly with the attenuated state to virulent strains by hyphal contact.

\section{RESULTS}

\section{Phenotypic characteristics of KFC9.}

Relative to the wild-type strains, the growth-rate of KFC9 was significantly reduced with progressive degeneration of the mycelium upon vegetative growth. The strain generally formed flat, highly pigmented cultures with irregular edges (Fig. 1). Conidiation was restricted to the center of the culture while the edges produced almost no aerial hyphae. Subcultures generated from mycelium taken from the center of degenerating cultures produced normal-appearing mycelia as they began to grow. However, as the hyphae advanced, growth became progressively thinner and the mycelia grew only within, rather than on the surface of, the agar medium. Growth always ceased before the edge of the petri dishes was reached. Because of the similarity of this process to the cytoplasmically transmitted, degenerative process described as "senescence" in other fungi, particularly Neurospora and Podospora spp., this term also has been adopted to describe this process in $C$. parasitica. Subcultures from degenerating mycelia were found to resume growth according to the stage of senescence present in the sampled region of the mycelium. That is, subcultures taken from the center of the senescing

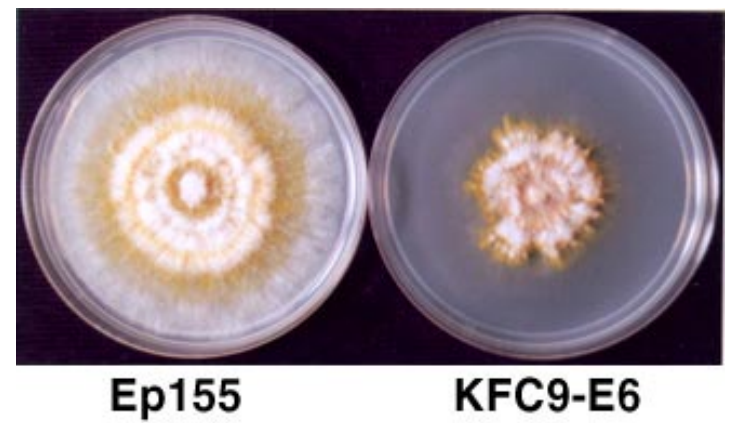

Fig. 1. Phenotypic and growth characteristics of the mutant KFC9-E6 and the wild-type Ep155. Both cultures are 7 days old. culture, where the mycelium appeared normal, exhibited the same pattern of growth and degeneracy as the parental culture, while subcultures started from plugs of mycelia taken progressively closer to the growth front showed increasingly advanced stages of senescence.

Cultures started from individual conidia from KFC9 also showed different degrees of senescence depending on the distance of their origin from the dying front of the parental culture. Conidia collected from the fast-growing center of a senescent culture generally demonstrated growth and debilitation patterns similar to those of the parental culture. However, conidia collected progressively from the center toward the edges formed colonies that showed increasing levels of degeneration. Some conidia produced only small colonies in which the mycelia grew very slowly before growth ceased.

\section{Serial transmission of the senescence phenotype through hyphal contact.}

To determine if nuclear or cytoplasmic factors were responsible for the senescence phenotype of KFC9, transmission studies were performed in which genetically marked, recipient, virulent strains were grown side by side with a donor hypovirulent strain. The leading edge of the recipient culture became very debilitated and formed appressed, thin mycelia that were morphologically similar to the degenerative phenotype of the leading edge of the donor mycelium. This degeneration then spread laterally through the leading edge of the recipient mycelium. Transmission of the senescence phenotype was accomplished serially, thereby demonstrating that infected recipients can also act as donors. The serial transmission of the senescence agent was conducted in the following order: from KFC9 to Ep289 to generate the hypovirulent strain Ep289[KFC9], from Ep289[KFC9] to A1.13 producing A1.13[KFC9], and then from A1.13[KFC9] to J2.31 generating the corresponding hypovirulent J2.31[KFC9] (Table 1). KFC9-E6 and the converted senescent form of Ep289 were used as donors with several other virulent recipient strains (Table 1). Conidia collected from Ep289[KFC9], a strain converted by KFC9, also showed the symptoms of senescence in some of the resulting colonies, thereby demonstrating that the effect of the senescence agent upon conidia is also transmitted between strains. Multiple attempts at extracting dsRNA failed to reveal the presence of any mycoviruses in any of the senescent strains (data not shown).

The extent of cytoplasmic transmissibility of the senescence phenotype across vegetative incompatibility barriers was also assessed by pairing the senescent KFC9 and KFC9-E6 strains, whose vegetative incompatibility (vic) genotype is unknown, with several tester strains with known vic genotypes. The

Table 1. Transmission of the 'senescence agent' by vegetative contact

\begin{tabular}{lll}
\hline Donor & Recipient $^{\mathbf{a}}$ & Senescent strain \\
\hline KFC9 & Ep289 met & Ep289[KFC9] met \\
Ep289[KFC9] met & C1.20 cre & C1.20[KFC9] cre \\
Ep289[KFC9] met & A1.13 cre & A1.13[KFC9] cre \\
A1.13[KFC9] cre & J2.31 br & J2.31[KFC9] br \\
KFC9-E6 & J2.31 br & J2.31[KFC9-E6] br \\
KFC9-E6 & F2.36 br & F2.36[KFC9-E6] $b r$ \\
\hline
\end{tabular}

${ }^{a}$ Recipients always differed from the donor by a nuclear marker as indicated: met $=$ methionine deficiency; $b r$ and $c r e=$ brown and cream color of mycelia, respectively. Wild-type pigmentation is orange. 
phenotype was found to be transmitted with high efficiency among compatible strains (Table 2). Transmission among incompatible strains was also observed and is particularly evident in the transmission of senescence from A1.13[KFC9] to $\mathrm{J} 2.31$, where the two strains differed only at locus 4, which has been found to be a weak inhibitor of transmission of dsRNA viruses (Huber 1996). The senescence phenotype was also found to be transmitted among incompatible strains that do not allow transmission of viruses among themselves. For example, donor Ep289[KFC9] and recipient strain A1.18 differed only at locus 1, with Ep289[KFC9] and A1.18 having alleles 1 and 2, respectively. Transmission of senescence was observed among these two strains despite the fact that transmission of viruses is restricted by this combination of donor and recipient vic genotypes (Huber 1996). Likewise, the senescent KFC9-E6 strain was able to convert two strains, F2.36 and $\mathbf{J} 2.31$, that are mutually incompatible because they differ at the strongest incompatibility locus, vic 2 (Huber 1996). The strain F2.36 has allele 1 at locus 2 while $\mathrm{J} 2.31$ has allele 2 in that position. However, since KFC9-E6 was able to infect both of these strains, it appears that the senescence phenotype can be transmitted even across strong incompatibility barriers. Nonetheless, it is clear that barriers to the transmission of the KFC9-type of hypovirulence exist between some strains. For example, the trait was not transferred from KFC9 to the strains 389.7, Ep388, 22508, F3.2, and F3.13 (Table 2). Since the compatibility genotype of KFC9 is unknown, it is unclear whether or not the lack of transfer was caused by allelic differences at vic loci or by other, yet undetermined factors that affect hyphal fusions. Collectively, the observations suggest that this novel senescence phenotype not only is cytoplasmically transmissible like dsRNA viruses, but also can be transmitted across barriers that prevent virus transmission between some highly incompatible strains.

\section{Respiration, senescence, and reduced virulence.}

Since the senescence and hypovirulence traits of KFC9 were transmitted asexually and independently of nuclei, experiments were conducted to determine the intracellular localization of the causative genetic-factor(s) for senescence. In

Table 2. Transmission of the 'senescence agent' across vegetative incompatibility barriers

\begin{tabular}{|c|c|c|c|c|}
\hline \multicolumn{2}{|c|}{ Donor } & \multicolumn{2}{|c|}{ Recipient } & \multirow{2}{*}{$\begin{array}{c}\text { Trans- } \\
\text { mission of } \\
\text { senescence } \\
\text { agent }\end{array}$} \\
\hline Strain & $v i c^{\mathrm{a}} 12345$ & Strain & $v i c^{\mathrm{a}} 12345$ & \\
\hline KFC9 & Unknown & 389.7 & 11111 & - \\
\hline KFC9 & Unknown & Ep388 & 21111 & - \\
\hline KFC9 & Unknown & Ep289 & 12111 & + \\
\hline KFC9 & Unknown & 22508 & 22111 & - \\
\hline KFC9 & Unknown & F3.2 & 11121 & - \\
\hline KFC9 & Unknown & F3.13 & 21121 & - \\
\hline KFC9-E6 & Unknown & $\mathrm{F} 2.36$ & 11111 & + \\
\hline KFC9-E6 & Unknown & $\mathrm{J} 2.31$ & 12121 & + \\
\hline Ep289[KFC9] & 12111 & $\mathrm{C} 1.3$ & 12111 & + \\
\hline Ep289[KFC9] & 12111 & A1.13 & 12111 & + \\
\hline Ep289[KFC9] & 12111 & A1.18 & 22111 & + \\
\hline A1.13[KFC9] & 12111 & $\mathrm{~J} 2.31$ & 12121 & + \\
\hline
\end{tabular}

a For brevity, vegetative incompatibility (vic) loci are designated by their number as a column heading. Alleles for each locus in a strain are listed in the column beneath the appropriate vic locus. Only two alleles, designated 1 and 2, are known for each vic locus (Huber 1996). filamentous fungi like Neurospora spp., Podospora anserina and Aspergillus amstelodami (reviewed in Griffiths 1992), Ophiostoma ulmi (Charter et al. 1993) and Cryphonectria parasitica (Monteiro-Vitorello et al. 1995), cytoplasmically transmissible phenotypes that induce slow growth and/or reduced virulence traits are typically associated with mitochondrial dysfunctions caused by mutant forms of mtDNA. Based on these observations, an attempt was made to establish whether or not the genetic agent that produces senescence in KFC9 is associated with mitochondria and might cause respiratory defects. Respiration was assayed in the senescent strains to determine if a defect in cytochrome-mediated respiration might be indicated by the presence of high levels of cyanide-resistant respiration (alternative oxidase activity).

The field-collected strains KFC9 and a subculture thereof, KFC9-E6, were found to have high levels of alternative oxidase activity (Table 3), whereas the activity in the wild-type strains of Ep289, C1.20, J2.31, and F2.36 was low and in the range of values for other virulent strains reported by Mahanti et al. (1993) and Monteiro-Vitorello et al. (1995). However, the converted senescent forms that were generated by hyphal contact of these virulent strains with KFC9 and KFC9-E6 exhibited high levels of cyanide-resistant respiration (61 to $88.5 \%$ ) (Table 3). Thus, it was evident that the senescence phenotype correlates with the transmission of a cytoplasmic factor capable of eliciting a mitochondrial dysfunction that induces the alternative respiratory pathway.

Virulence of the wild-type and senescent strains was measured by the size of lesions produced on apples, as described by Fulbright (1984), and/or on live chestnut tissue by the method of Lee et al. (1992). The virulence level of KFC9-E6, a subculture of the wild-collected KFC9 strain, was found to be very low (Table 3 ). The virulence of all of the wild-type strains was found to be drastically reduced when they were infected with the senescence agent. In fact, the strains Ep289 [KFC9], J2.31[KFC9], and F2.36[KFC9] often failed to grow when inoculated into apples or chestnut bark. Hence, these

Table 3. Alternative oxidase (AO) activity as percentage of total respiration (\% tot. resp.) and virulence of wild-type and corresponding senescent strains cytoplasmically infected with the senescence-inducing agent from KFC9 and KFC9-E6

\begin{tabular}{lcccc}
\hline Strain & $\begin{array}{c}\text { Pheno- } \\
\text { type }\end{array}$ & $\begin{array}{c}\text { Virulence on } \\
\text { chestnut } \\
\text { tissue }^{\mathbf{a}}\end{array}$ & $\begin{array}{c}\text { Virulence on } \\
\text { apple }^{\mathbf{a}}\end{array}$ & $\begin{array}{c}\text { AO (\% tot. } \\
\text { resp.) }\end{array}$ \\
\hline Ep155 & $\mathrm{N}^{\mathrm{b}}$ & $\mathrm{NT}^{\mathrm{c}}$ & $21.1 \pm 2.0$ & $7.5 \pm 0.3$ \\
KFC9 & $\mathrm{S}^{\mathrm{d}}$ & $\mathrm{NT}^{\mathrm{e}}$ & $\mathrm{NT}^{\mathrm{e}}$ & $78.0 \pm 10.4$ \\
KFC9-E6 & $\mathrm{S}$ & $\mathrm{NT}$ & $1.0 \pm 1.1$ & $38.6 \pm 7.8$ \\
Ep289 & $\mathrm{N}$ & $4.2 \pm 0.2$ & $\mathrm{NT}$ & $21.3 \pm 2.7$ \\
Ep289[KFC9] & $\mathrm{S}$ & $0.2 \pm 0.1$ & $\mathrm{NT}$ & $81.7 \pm 1.1$ \\
C1.20 & $\mathrm{N}$ & $7.0 \pm 0.1$ & $\mathrm{NT}$ & $14^{\mathrm{f}}$ \\
C1.20[KFC9] & $\mathrm{S}$ & $1.3 \pm 0.3$ & $\mathrm{NT}$ & $61^{\mathrm{f}}$ \\
J2.31 & $\mathrm{N}$ & $6.4 \pm 0.4$ & $17.3 \pm 2.0$ & $13.1 \pm 3.3$ \\
J2.31[KFC9] & $\mathrm{S}$ & $0.3 \pm 0.2$ & $<1.0$ & $80.5 \pm 4.2$ \\
J2.31[KFC9-E6] & $\mathrm{S}$ & $\mathrm{NT}$ & $<1.0$ & $88.5 \pm 3.1$ \\
F2.36 & $\mathrm{N}$ & $\mathrm{NT}$ & $13.2 \pm 1.4$ & $10.5 \pm 6.0$ \\
F2.36[KFC9-E6] & $\mathrm{S}$ & $\mathrm{NT}$ & $<1.0$ & $68.4 \pm 9.6$ \\
\hline
\end{tabular}

${ }^{\text {a }}$ Virulence measured as area of lesion in $\mathrm{cm}^{2}$.

b Nonsenescent strain.

c Not tested.

d Senescent strain.

e Strain lost to senescence before virulence tests could be performed.

${ }^{\mathrm{f}}$ Observation based on one sample. 
strains are afflicted with a disease syndrome that can be referred to as "mitochondrial hypovirulence" as defined by Monteiro-Vitorello et al. (1995).

\section{Identification of the segment of mtDNA bearing the senescence agent.}

The mtDNAs isolated from purified mitochondria of KFC9E6, Ep289 and Ep289[KFC9] were digested with HindIII and the resulting fragments were separated by agarose gel electrophoresis. The restriction digestion patterns of the mtDNAs of KFC9-E6 and Ep289 differed by multiple restriction fragment length polymorphisms (Fig. 2A). In contrast, Ep289 differed from Ep289[KFC9] in the size of only one fragment; i.e., a $10.5-\mathrm{kb}$ band in Ep289 was replaced by an 11.5-kb band in Ep289[KFC9]. This 11.5-kb fragment appeared to have originated from the mtDNA of KFC9, which produces it, and not the 10.5-kb fragment when digested with HindIII. That the nuclei of the recipient remained unaltered during its infection by KFC9 was evident because the nuclear DNA fingerprints of Ep289 and Ep289[KFC9] were found to be identical to each other and different from that of KFC9-E6 (Fig. 3). The 11.5-kb HindIII fragment of mtDNA also appeared in the converted $\mathbf{J} 2.31$ [KFC9], whereas restriction of the mtDNA for the virulent $\mathrm{J} 2.31$ strain produced only the $10.5-\mathrm{kb}$ HindIII fragment (Fig. 2B). The displacement of the 10.5-kb HindIII fragment by an 11.5-kb fragment also was associated with the transfer of the hypovirulence trait from KFC9-E6 to the wildtypes F2.36 and J2.31 (data not shown). Since this was the only DNA fragment that correlated with the transmission of the senescence phenotype, the 11.5-kb fragment was hypothesized to contain the KFC9 senescence-agent. The fact that the converted hypovirulent recipients retained most of the mtDNA of their virulent progenitors further supports the argument that the $11.5-\mathrm{kb}$ fragment includes the genetic factor that causes the senescence phenotype and hypovirulence traits
A

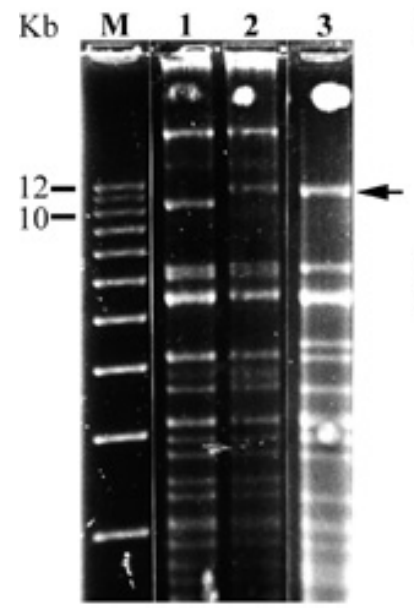

B

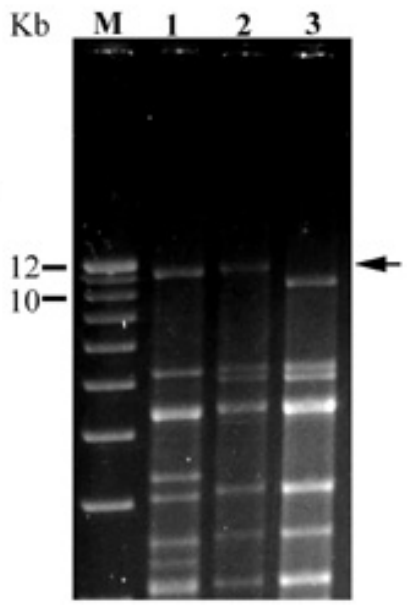

Fig. 2. Identification of region of mitochondrial DNA (mtDNA) bearing putative mutation in KFC9-E6. HindIII-digested mtDNA was fractionated by agarose gel electrophoresis and visualized by ethidium bromide staining. Invasive HindIII fragment bearing senescence-inducing mutation is indicated by arrows. A, Lane 1, Ep289; lane 2, Ep289[KFC9]; lane 3, KFC9-E6. B, Lane 1, KFC9-E6; lane 2, J2.31[KFC9-E6]; lane 3, J2.31. A and $\mathbf{B}$, Lane $\mathbf{M}=$ molecular weight markers. found in KFC9. The putative mutation associated with this segment of the mtDNA was mapped and identified as a 973bp insert that is located in the mitochondrial small subunit ribosomal RNA (SSUrRNA) gene and lacks features that would identify it as either an intron or a mobile genetic element. The nucleotide sequence of this insert and its effect on the processing of the precursor of the mitochondrial SSUrRNA will be published elsewhere. All of the converted hypovirulent strains were found to contain the mutant form of the SSUrRNA gene. The corresponding segment of their mtDNA was amplified in a polymerase chain reaction (PCR) with primers that generate a 700-bp fragment from wild-type virulent strains and a 1.7-kb fragment in strains that have the KFC9 senescence phenotype (Fig. 4). However, some of the converted hypovirulent strains were occasionally found to be heteroplasmic, as indicated by amplification of both the wild-type and the mutant forms of the mtDNA in PCRs (Fig. 4, lane 3). Contamination of the PCR mixtures was ruled out because similar products were always obtained from reactions with the same preparation of DNA as the template.

\section{Dissemination of mitochondrial hypovirulence in nature.}

Several strains were collected from trees located in the area of origin of KFC9, and genomic DNA was prepared from each isolate. These DNAs were used as templates for the amplification of the SSUrRNA region that is affected in KFC9 by

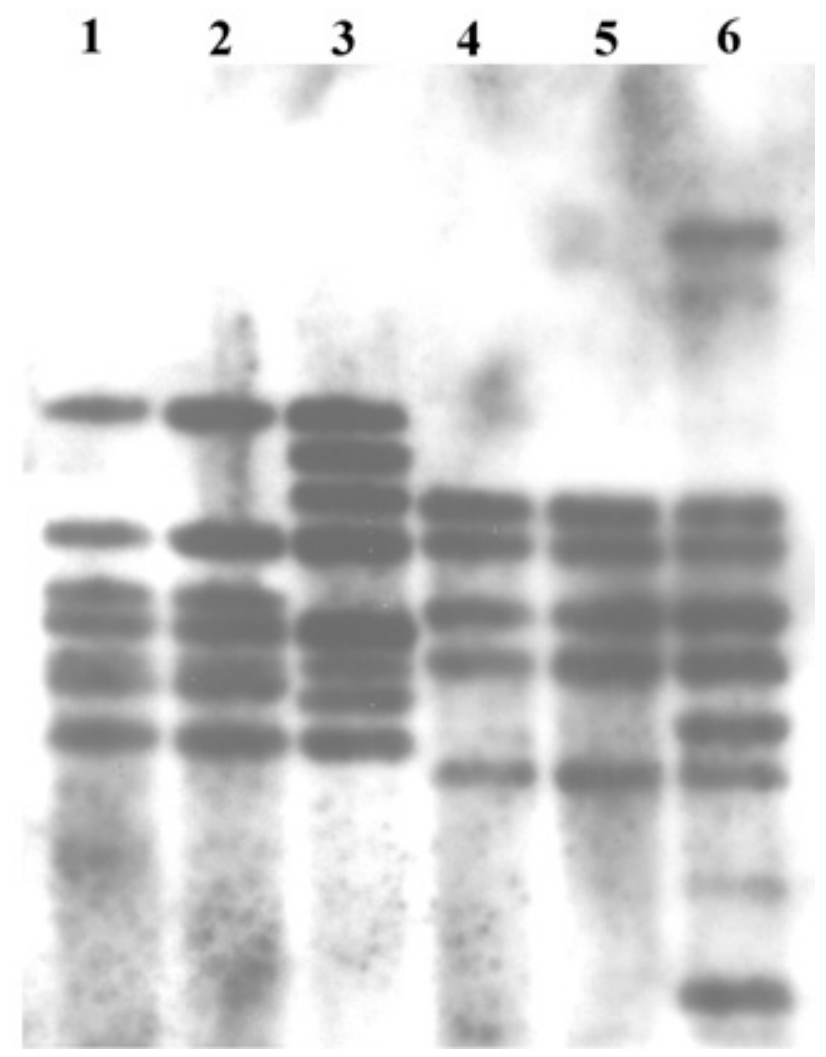

Fig. 3. Comparison of nuclear genotypes of the wild-type strain Ep289, converted hypovirulent strain Ep289[KFC9], and hypovirulent strain KFC9. Genomic DNA isolated from the strains was digested with HincII (lanes 1-3) and HindIII (lanes 4-6) and fractionated on an agarose gel. DNA from the gel was transferred to a nylon membrane and hybridized with the fingerprinting probe pMS5.1. Lanes 1 and 4, Ep289; lanes 2 and 5, Ep289[KFC9]; lanes 3 and 6, KFC9. 
PCR with primers that flank the putative insertion. In the strains that were included in this experiment, only one was found that did not contain the putative senescence agent (Fig. $5)$. This implies that the agent that causes hypovirulence and senescence in KFC9 occurs in a large proportion of the $C$. parasitica population at that location. Only the strain that did not contain the senescence agent (KFD19.2) was found to be virulent and did not show high levels of cyanide-resistant respiration (data not shown). Among 13 strains collected in 1997, 12 were moderately to severely hypovirulent (Table 4). In contrast, out of 19 strains collected from this site in 1990, only six demonstrated any degree of hypovirulence. None of these strains were found to contain any dsRNA virus. The data in Table 4 indicate that there has been a marked shift in the ratio of hypovirulent to virulent strains from 1990 to 1997, suggesting that the mtDNA that causes this trait is probably invading the population of $C$. parasitica at the Kellogg Forest site.

To explore whether or not the strains isolated from the Kellogg Forest site represent a genetically homogenous population, nuclear genotypes were determined with a probe for repetitive DNA, as described by Milgroom et al. (1992).

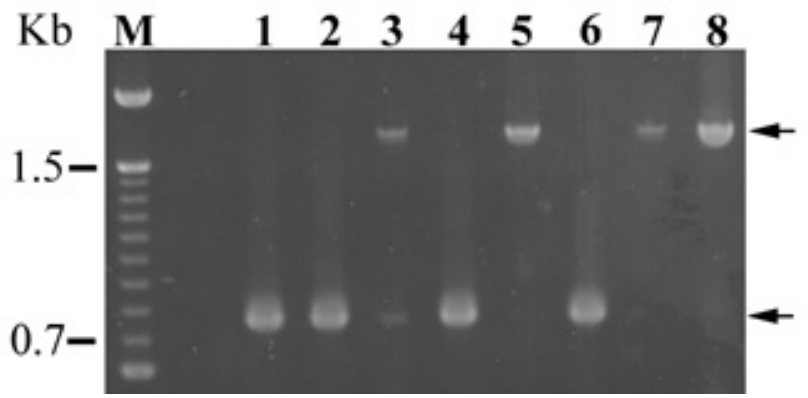

Fig. 4. Conversion of wild-type mtDNA into mutant form in converted hypovirulent strains as detected by polymerase chain reaction (PCR). Products generated from PCR were separated by agarose gel electrophoresis and observed by ethidium bromide staining. Lane 1, Ep155; lane 2, Ep289; lane 3, Ep289[KFC9]; lane 4, J2.31; lane 5, J2.31[KFC9]; lane 6, F2.36; lane 7, F2.36[KFC-E6]; lane 8, KFC9-E6. Lane M, the molecular weight marker. The $0.7-$ and $1.7-\mathrm{kb}$ fragments indicated by arrows. DNA bearing the putative mutation is approximately $1 \mathrm{~kb}$ larger than the wild-type fragment.

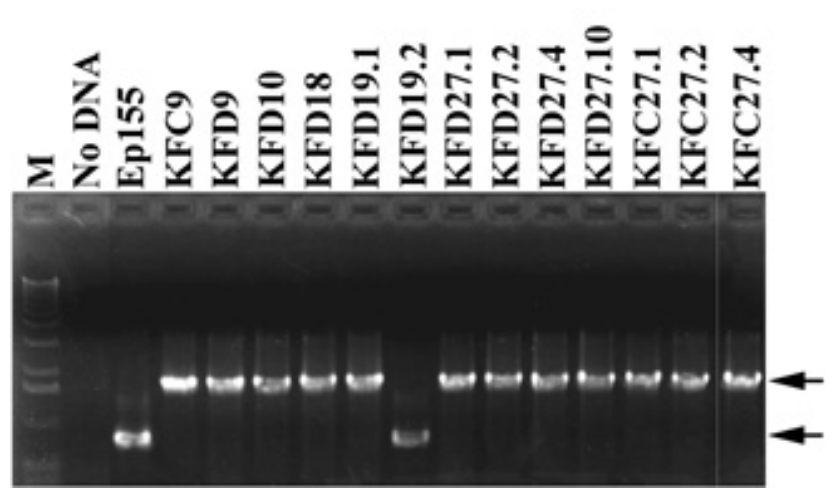

Fig. 5. Dissemination of the mutation of KFC9 in nature as detected by polymerase chain reaction (PCR). PCR products were separated by agarose gel electrophoresis and visualized by UV fluorescence after ethidium bromide staining. The $0.7-$ and $1.7-\mathrm{kb}$ fragments are indicated by arrows. Lane $\mathrm{M}=$ the molecular weight marker.
Out of the 12 strains included in this experiment, eight unique patterns of hybridization were identified (Fig. 6). Strains isolated from the same tree were often found to have different nuclear genotypes. For example, strains KFD19.1 and KFD19.2 originated from the same tree (number 19), but had different DNA fingerprint patterns. The same was true for strains KFC9 and KFD9 from tree 9 and strains KFD27.1, KFD27.2, KFD27.4, and KFD27.10 from tree 27. In contrast, some of the strains from different trees were found to have similar nuclear genotypes, e.g., KFC9 (tree 9), KFD18 (tree 18) and KFD27.2 (tree 27). Although relatively few strains were included in this study, different strains from the same canker were always found to be similar, for example KFD27.2 and KFC27.2 (tree 27, canker 2) and KFD27.4 and KFC27.4 (tree 27, canker 4). Several highly debilitated strains could not be included in this analysis because they died before DNA could be extracted from their mycelia. None of these strains contained a detectable amount of any dsRNA virus. Collectively, these observations suggest that the hypovirulence factor may be transmitted asexually through hyphal anastomoses between mycelia of different genotypes or disseminated sexually by maternal inheritance in crosses between different strains.

\section{DISCUSSION}

In this study, we provide a characterization and a brief analysis of the dispersal of a novel mitochondrial hypovirulence and senescence syndrome that has appeared spontaneously in $C$. parasitica on American chestnut trees. The study describes a novel type of hypovirulence in which the afflicted

Table 4. Progression of hypovirulence in Cryphonectria parasitica strains at Kellogg Forest in Augusta, MI

\begin{tabular}{lccc}
\hline Virulence status & $\begin{array}{c}\text { Area of lesion produced } \\
\text { on apples in } \mathbf{~ c m}^{\mathbf{2}}\end{array}$ & \multicolumn{2}{c}{ Strains collected } \\
\cline { 3 - 4 } & $>15$ & $\mathbf{1 9 9 0}$ & $\mathbf{1 9 9 7}$ \\
\hline Virulent & $8-15$ & 2 & 1 \\
Moderate & $<8$ & 4 & 3 \\
Hypovirulent & & \\
\hline
\end{tabular}

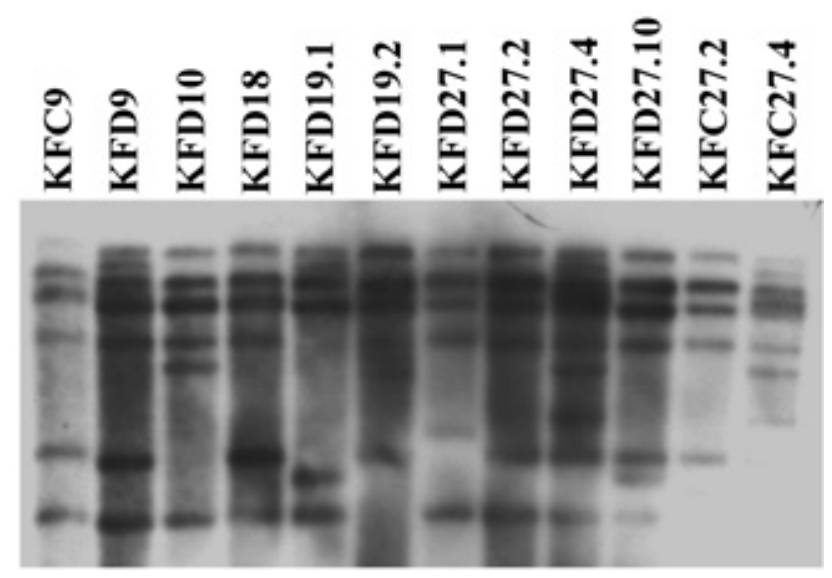

Fig. 6. Nuclear genotypes of strains obtained from the Kellogg Forest area. Genomic DNA isolated from the strains was digested with PstI and separated on an agarose gel. DNA from the gel was transferred to a nylon membrane and hybridized with the fingerprinting probe pMS5.1. 
strains progressively degenerate during vegetative growth and demonstrate elevated levels of alternative oxidase activity. The association of mtDNA mutations with this senescence syndrome is indicated by the nonsynchronous germination of conidia, cytoplasmic transmission of the senescence phenotype, and co-transfer of a unique segment of mtDNA from donor to recipient strains during transmission of the senescence factor. The aberrant germination of the conidia from KFC9 can be explained by random distribution of mutant and normal mitochondria from heteroplasmic hyphae into the asexual spores, resulting in fast germination of conidia containing few mutant mitochondria and slower germination of the ones that contain high amounts of dysfunctional mitochondria. These characteristics are similar to the transmissible senescence syndromes of Neurospora spp. that are caused by suppressive mtDNA mutations producing dysfunctional mitochondria that proliferate more rapidly than normal mitochondria and thus invade the coenocytic mycelia of this organism (Bertrand et al. 1986; Bertrand 1995).

In many filamentous fungi, senescence has been shown to be associated with respiratory defects that are caused by a variety of mtDNA mutations. Excision, circularization, and subsequent amplification of small segments of the mitochondrial chromosome are observed in senescent strains of Podospora anserina (Jamet-Vierney et al. 1980), Aspergillus amstelodami (Lazarus et al. 1980), and Ophiostoma ulmi (Abu Amero et al. 1995). Amplification of segments of the mitochondrial chromosome also has been observed in the mitochondria of mitochondrially hypovirulent $C$. parasitica strains (MonteiroVitorello et al. 1995). Large deletions in the mtDNA have been found in senescent stopper mutants of $N$. crassa (Bertrand et al. 1980) as well as in the ragged mutants of Aspergillus amstelodami (Lazarus and Küntzel 1981) and in senescent cultures of Podospora curvicolla (Böckelmann and Esser, 1986) as well as P. anserina (Contamine et al. 1996). Small deletions (Manella and Lambowitz 1978) and integration of plasmids into the mtDNA (Bertrand et al. 1986; Akins et al. 1986) can also lead to senescence in Neurospora spp. Therefore, in the case of the KFC9-type of senescence in $C$. parasitica, it is plausible that the 973-bp DNA insert in the SSUrRNA gene causes the debilitation of the fungus. However, the possibility that the phenotype is caused by a point mutation that is closely linked to this insert or exists elsewhere in a nonpolymorphic region of the mtDNA cannot be excluded at this time. Since a variety of mtDNA mutations can cause senescence or suppressive slow-growth phenotypes, it seems that it is the final physiological effect of these mutations, namely respiratory deficiency, that causes suppressiveness, rather than the individual mutations themselves (Bertrand 1995).

The concomitant transfer of an insertion in the mtDNA of KFC9 with the senescence and hypovirulence phenotypes potentially could represent any of the following three situations. First, the insertion could be a stable, deleterious mutation in the SSUrRNA that causes the suppressive accumulation of defective mtDNA molecules upon its transfer into unaffected strains, thereby causing senescence. In this case, the mechanism for the transfer of the insert into the mtDNA of recipient strains and the disappearance of the mtDNA from the donors remains unexplained. Secondly, the insertion could be a sitespecific, mobile, genetic element that spreads by inserting into the SSUrRNA gene, thus converting normal mtDNA into the mutant form. In this case, senescence would be a reflection of the conversion process, but the insert lacks features identifying it as a transposable element. Finally, the insertion could be a homing intron that moves into intron-less copies of the SSUrRNA gene. However, senescence would not be expected in this case because the intronic sequence most likely would be spliced from the rRNA primary transcript. On the basis of information available about senescence in other fungi, the first and the third options are the most and the least likely, respectively, of the mechanisms that could account for senescence in strains that have the KFC9 cytoplasm. The precise identification of the molecular and physiological processes that are involved in the progressive attenuation of the population of $C$. parasitica at the Kellogg Forest site requires further experimentation.

Since the presence of the $11.5-\mathrm{kb}$ HindIII fragment in the mtDNA of different strains correlates with the appearance of the hypovirulence phenotype (Fig. 2), the screening of a sample of isolates from trees in the Kellogg Forest for this DNA segment by PCR can be used to assess the extent and progress of the dissemination of the KFC9 type of hypovirulence at that location. Among all of the isolates recovered from the Kellogg Forest site in 1997, only one strain did not contain the mutant mtDNA (Fig. 5). This strain was found to be virulent and does not demonstrate elevated levels of alternative oxidase activity. Due to the nuclear heterogeneity present among these strains (Fig. 6), it can be assumed that not all of them are vegetatively compatible with each other. Hence, the KFC9-senescence phenotype not only might be transmitted vegetatively through hyphal anastomoses, but also may be sexually inherited. In $C$. parasitica, it has been shown previously that some debilitating mtDNA mutations indeed can be sexually inherited (Monteiro-Vitorello et al. 1995). This particular feature of mitochondrial hypovirulence is relevant because Cryphonectria strains are known to be cured of dsRNA hypoviruses during the sexual cycle (Nuss 1992). Asexual transmission of the phenotype among incompatible strains demonstrates that, like viruses, mitochondrial hypovirulence can be transmitted efficiently through hyphal contacts. It has been found that vegetative incompatibility loci sometimes may reduce the rate of, and even block, the asexual transmission of mitochondrial mutations (Caten 1972; and Table 2 in this study). However, the observations presented in Figure 5 suggest that the dynamics of the dissemination of the KFC9 factor in a genetically heterogeneous population of $C$. parasitica that exists in a natural environment may be more liberal than that which may be deduced from laboratory studies.

The presence of the senescent factor in most of the strains from Kellogg Forest also implies that the KFC9 type of hypovirulence is not only highly transmissible, but also viable and stably maintained in nature and can infect a substantial proportion of virulent strains in a population of the fungus. This observation is consistent with the finding that most of the infected trees in the Kellogg Forest are now recovering. Collectively, these results indicate that mitochondrial hypovirulence in $C$. parasitica might be used effectively instead of, or in addition to, dsRNA viruses in the biological control of this fungus. Since a majority of plant diseases are caused by fungi, the discovery and analyses of mtDNA mutations and other deleterious mitochondrial genetic elements could significantly impact the development of effective biological control meth- 
ods. In this context, the findings of this study suggest that the dissemination of hypovirulent strains containing mtDNA mutations might be useful as a remedial measure against fungal diseases and potentially can be applied to control virulent populations of a variety of phytopathogenic fungi.

\section{MATERIALS AND METHODS}

Fungal strains, culturing conditions, respiration assays, and virulence tests.

The strains of $C$. parasitica used in this study are listed in Table 5. Cultures were grown in Endothia complete medium (ECM) as described (Puhalla and Anagnostakis 1971) or on potato dextrose agar (PDA; Difco Laboratories, Detroit, MI). Tests for alternative oxidase activity were performed as described by Monteiro-Vitorello et al. (1995). Virulence tests were performed either on apples (Fulbright 1984) or on live chestnut tissue (Lee et al. 1992).

\section{Transmission of hypovirulence phenotype through hyphal contact.}

Mycelial plugs of the donor and recipient strains were placed side by side, about $0.5 \mathrm{~cm}$ apart, on ECM near the walls of petri dishes and were allowed to grow until the cultures reached the opposite sides of the plates. Small mycelial plugs from the periphery of cultures of the recipient strain were then taken and subcultured on fresh plates. The subcul-

Table 5. Strains of Cryphonectria parasitica used in this study

\begin{tabular}{|c|c|c|}
\hline Strain & Senescence agent & Source \\
\hline Ep155 & None & ATCC 38755 \\
\hline KFC9 & Present & Kellogg Forest, MI \\
\hline KFC9-E6 (KFC9 subculture) & Present & Kellogg Forest, MI \\
\hline KFD9 & Present & Kellogg Forest, MI \\
\hline KFD10 & Present & Kellogg Forest, MI \\
\hline KFD18 & Present & Kellogg Forest, MI \\
\hline KFD19.1 & Present & Kellogg Forest, MI \\
\hline KFD19.2 & None & Kellogg Forest, MI \\
\hline KFD27.1 & Present & Kellogg Forest, MI \\
\hline KFC27.1 & Present & Kellogg Forest, MI \\
\hline KFD27.2 & Present & Kellogg Forest, MI \\
\hline KFC27.2 & Present & Kellogg Forest, MI \\
\hline KFC27.3 & Present & Kellogg Forest, MI \\
\hline KFD27.4 & Present & Kellogg Forest, MI \\
\hline $\mathrm{KFC} 27.4$ & Present & Kellogg Forest, MI \\
\hline KFD27.10 & Present & Kellogg Forest, MI \\
\hline Ep289 & None & Conn. Agri. Exp. ${ }^{a}$ \\
\hline Ep289[KFC9] & Present & This study \\
\hline $\mathrm{J} 2.31$ & None & DWF collection ${ }^{b}$ \\
\hline $\mathrm{J} 2.31[\mathrm{KFC} 9]$ & Present & This study \\
\hline $\mathrm{F} 2.36$ & None & DWF collection \\
\hline F2.36[KFC9] & Present & This study \\
\hline A 1.13 & None & DWF collection \\
\hline A1.13[KFC9] & Present & This study \\
\hline A1.18 & None & DWF collection \\
\hline $\mathrm{C} 1.20$ & None & DWF collection \\
\hline C1.20[KFC9] & Present & This study \\
\hline $\mathrm{C} 1.3$ & None & DWF collection \\
\hline 389.7 & None & DWF collection \\
\hline 22508 & None & ATCC 22508 \\
\hline Ep388 & None & ATCC 38979 \\
\hline F3.2 & None & DWF collection \\
\hline F3.13 & None & DWF collection \\
\hline
\end{tabular}

${ }^{\mathrm{a}}$ Sandra Anagnostakis, Connecticut Agricultural Experiment Station.

${ }^{b}$ Dennis W. Fulbright collection, Michigan State University. turing was repeated until the recipient demonstrated the senescence phenotype, which normally occurred between two and five transfers. Virulent strains that have acquired hypovirulence by hyphal contact are mentioned by their strain name followed by "[KFC9]" to denote that they have a cytoplasmic factor derived from the strain KFC9.

\section{Isolation of genomic and mitochondrial DNA.}

For genomic DNA preparations, mycelia were grown overnight in $500 \mathrm{ml}$ of ECM broth while being shaken at $200 \mathrm{rpm}$ in a G10 gyratory shaker (New Brunswick Scientific, Edison, $\mathrm{NJ}$ ). Approximately 3 to $5 \mathrm{~g}$ of each mycelium was collected by filtration and then disrupted by grinding with acid-washed sand (Fisher Scientific, Pittsburgh, PA) in the cold. The disrupted hyphae were suspended in 8 to $10 \mathrm{ml}$ of a solution containing $0.2 \%$ sodium dodecyl sulfate and $0.1 \mathrm{M}$ EDTA and the homogenate was incubated at $70^{\circ} \mathrm{C}$ for $15 \mathrm{~min}$. Then, $2 \mathrm{ml}$ of $5 \mathrm{M}$ potassium acetate was added to the tubes. The solutions were mixed gently and incubated for $1 \mathrm{~h}$ on ice. The mixture was centrifuged at $14,000 \mathrm{rpm}$ for $10 \mathrm{~min}$ in a Sorvall SS-34 rotor (DuPont, Wilmington, DE). The supernatant was collected carefully and DNA was precipitated with an equal volume of isopropanol. The DNA was redissolved in $0.5 \mathrm{ml}$ of TE buffer (10 mM Tris-HCL, $1 \mathrm{mM}$ EDTA, pH 7.6) supplemented with RNaseA $(10 \mu \mathrm{g} / \mathrm{ml})$ and incubated at $37^{\circ} \mathrm{C}$ for 30 to $45 \mathrm{~min}$. The DNA was further purified by successive phenol, phenol:chloroform, and chloroform extractions. In some cases, the DNA was also purified with cetyltrimethylammoniumbromide (CTAB; USB, Cleveland, OH) (Ausubel et al. 1987). Mitochondrial DNA was purified as described by Bell et al. (1996) with an added purification step with CTAB.

\section{DNA manipulations and Southern blot hybridization.}

Restriction enzymes were obtained from Gibco BRL (Gaithersburg, MD). Enzymatic digestions of DNAs, agarose gel electrophoresis, and Southern blotting were done as described by Sambrook et al. (1989). Southern blot hybridizations were performed with chemiluminescent probes as directed by the manufacturer (Boehringer Mannheim, Indianapolis, IN). For nuclear DNA fingerprinting, Southern blots were hybridized with a plasmid (pMS5.1) containing a cloned, moderately repetitive, nuclear DNA fragment (Milgroom et al. 1992).

\section{PCR.}

PCR was done as suggested by the manufacturer (Promega, Madison, WI). Template DNAs were boiled for 5 min before being added to the reaction tubes. The DNA was denatured at $93^{\circ} \mathrm{C}$ for 3 min and subjected to PCR for 30 cycles, each consisting of the following successive steps: $93^{\circ} \mathrm{C}$ for $1 \mathrm{~min}$, $52^{\circ} \mathrm{C}$ for $1 \mathrm{~min}$, and $72^{\circ} \mathrm{C}$ for $1 \mathrm{~min}$. The last cycle consisted of an extension reaction at $72^{\circ} \mathrm{C}$ for $10 \mathrm{~min}$. The sequence of the primers used in these reactions was designed on the basis of the nucleotide sequence of the SSUrRNA gene of Cryphonectria parasitica (GenBank accession no. AF029891) and is as follows: 5'-GGTTGGTGATTCTTTCATGG-3' (forward primer) and 5'-TACACTCACCTGTACAC-3' (reverse primer).

\section{ACKNOWLEDGMENTS}

This work was supported in part by USDA grant 95-37303-1785 and Michigan Agriculture Experiment Station Project No. MICL01662. A 
Sigma Xi grant in aid of research to D. B. and support from the NSF Center for Microbial Ecology to D. H. are gratefully acknowledged. We wish to thank Georg Hausner and Claudia Monteiro-Vitorello for their help and advice and Jonathan D. Walton for the critical review of the manuscript.

\section{LITERATURE CITED}

Abu-Amero, S. N., Charter, N. W., Buck, K. W., and Brasier, C. M. 1995. Nucleotide sequence analysis indicates that a DNA plasmid in a diseased isolate of Ophiostoma novo-ulmi is derived by recombination between two long repeat sequences in the mitochondrial large subunit ribosomal RNA gene. Curr. Genet. 28:54-59.

Akins, R. A., Kelley, R. L., and Lambowitz, A. M. 1986. Mitochondrial plasmids of Neurospora: integration into mitochondrial DNA and evidence for reverse transcription in mitochondria. Cell 47:505-516.

Ausubel, F., Brent, R., Kingston, R. E., Moore, D. D., Seidman, J. G., Smith J. A., and Struhl, K. 1987. Current Protocols in Molecular Biology. John Wiley and Sons, New York.

Bell, J. A., Monteiro-Vitorello, C. B., Hausner, G., Fulbright, D. W., and Bertrand, H. 1996. Physical and genetic map of the mitochondrial genome of Cryphonectria parasitica Ep155. Curr. Genet. 30:34-43.

Bertrand, H. 1995. Senescence is coupled to induction of an oxidative phosphorylation stress response by mitochondrial DNA mutations in Neurospora. Can. J. Bot. 73 (Suppl. 1):S198-S204.

Bertrand, H., Collins, R. A., Stohl, L. L., Goewert, R., and Lambowitz, A. M. 1980. Deletion mutants of Neurospora crassa mitochondrial DNA and their relationship to the "stopper" growth phenotype. Proc. Natl. Acad. Sci. USA 77:6032-6036.

Bertrand, H., Griffiths, A. J. F., Court, D. A., and Cheng, C. K. 1986. An extrachromosomal plasmid is the etiological precursor of kalDNA insertion sequences in the mitochondrial chromosome of senescent $\mathrm{Neu}$ rospora. Cell 47:829-837.

Böckelmann, B., and Esser, K. 1986. Plasmids of mitochondrial origin in senescent mycelia of Podospora curvicolla. Curr. Genet. 10:803-810.

Brasier, C. M. 1983. A cytoplasmically transmitted disease of Ceratocystis ulmi. Nature 305:220-223.

Buck, K. W. 1986. Fungal virology - an overview. Pages 2-84 in: Fungal Virology. K. W. Buck, ed. CRC, Boca Raton, FL.

Caten, C. E. 1972. Vegetative incompatibility and cytoplasmic infection in fungi. J. Gen. Microbiol. 72:221-229.

Charter, N. W., Buck, K. W., and Brasier, C. M. 1993. De-novo generation of mitochondrial-DNA plasmids following cytoplasmic transmission of a degenerative disease in Ophiostoma novo-ulmi. Curr. Genet. 24:505-514.

Contamine, V., Lecellier, G., Belcour, L., and Picard, M. 1996. Premature death in Podospora anserina: Sporadic accumulation of the deleted mitochondrial genome, translational parameters and innocuity of the mating types. Genetics 144:541-555.

Fulbright, D. W. 1984. Effect of eliminating dsRNA in hypovirulent Endothia parasitica. Phytopathology 74:722-724.

Ghabrial, S. A. 1988. Viruses of Helminthosporium victoriae. Pages 353-369 in: Viruses of Fungi and Lower Eukaryotes. Y. Koltin and M. J. Leibowitz, eds. Marcel Dekker, New York.

Griffiths, A. J. F. 1992. Fungal senescence. Annu. Rev. Genet. 26:351-372.

Huber, D. H. 1996. Genetic analysis of vegetative incompatibility polymorphisms and horizontal transmission in the chestnut blight fungus,
Cryphonectria parasitica. Ph.D. diss. Michigan State University, East Lansing.

Jamet-Vierney, C., Begel, O., and Belcour, L. 1980. Senescence in Podospora anserina: amplification of a mitochondrial DNA sequence. Cell 21:189-194.

Lakshman, D. K. and Tavantzis, S. M. 1994. Spontaneous appearance of genetically distinct double stranded-RNA elements in Rhizoctonia solanii. Phytopathology 84:633-639.

Lambowitz, A. M., and Slayman, C. W. 1971. Cyanide-resistant respiration in Neurospora crassa. J. Bacteriol. 108:1087-1096.

Lambowitz, A. M., and Zannoni, D. 1978. Cyanide-insensitive respiration in Neurospora. Genetic and biophysical approaches. Pages 283291 in: Plant Mitochondria. G. Ducet and C. Lance, eds. Elsevier/North-Holland Biomedical, Amsterdam.

Lazarus, C. M., Earl, A. J., Turner, G., and Küntzel, H. 1980. Amplification of a mitochondrial DNA sequence in the cytoplasmically inherited 'ragged' mutant of Aspergillus amstelodami. Eur. J. Biochem. 106:633-641.

Lazarus, C. M., and Küntzel, H. 1981. Anatomy of amplified mitochondrial DNA in 'ragged' mutants of Aspergillus amstelodami: excision points within protein genes and a common $215 \mathrm{bp}$ segment containing a possible origin of replication. Curr. Genet. 4:99-107.

Lee, J. K., Tattar, T. A., Berman, P. M., and Mount, M. S. 1992. A rapid method for testing the virulence of Cryphonectria parasitica using excised bark and wood of American chestnut. Phytopathology 82: 1454-1456.

Mahanti, N., Bertrand, H., Monteiro-Vitorello, C. B., and Fulbright, D. W. 1993. Elevated mitochondrial alternative oxidase activity in dsRNA-free, hypovirulent isolates of Cryphonectria parasitica. Physiol. Mol. Plant Pathol. 42:455-463.

Manella, C. A., and Lambowitz, A. M. 1978. Interaction of wild-type and poky mitochondrial DNA in heterokaryons of Neurospora. Biochem. Biophys. Res. Commun. 80:673-679.

Milgroom, M. G., Lipari, S. E., and Powell, W. A. 1992. DNA fingerprinting and analysis of population structures of the chestnut blight fungus Cryphonectria parasitica. Genetics 131:297-306.

Monteiro-Vitorello, C. B., Bell, J. A., Fulbright, D. W., and Bertrand, H. 1995. A cytoplasmically transmissible hypovirulence phenotype associated with mitochondrial DNA mutations in the chestnut blight fungus Cryphonectria parasitica. Proc. Natl. Acad. Sci. USA 92:59355939.

Nuss, D. L. 1992. Biological control of chestnut blight: An example of virus-mediated attenuation of fungal pathogenesis. Microbiol. Rev. 56:561-576.

Nuss, D. L. 1996. Using hypoviruses to probe and perturb signal transduction processes underlying fungal pathogenesis. Plant Cell 8:18451853.

Puhalla, J. E., and Anagnostakis, S. L. 1971. Genetics and nutritional requirements of Endothia parasitica. Phytopathology 61:169-173.

Rogers, H. J., Buck, K. W., and Brasier, C. M. 1986. Transmission of double-stranded RNA and a disease factor in Ophistoma ulmi. Plant Pathol. 35:277-287.

Sambrook, J., Fritsch, E. F., and Maniatis, T. A. 1989. Molecular Cloning: A Laboratory Manual. 2nd ed. Cold Spring Harbor Laboratory, Cold Spring Harbor, NY.

Vanlerberghe, G. C., and McIntosh, L. 1997. Alternative oxidase: From gene to function. Annu. Rev. Plant Physiol. 48:703-734. 